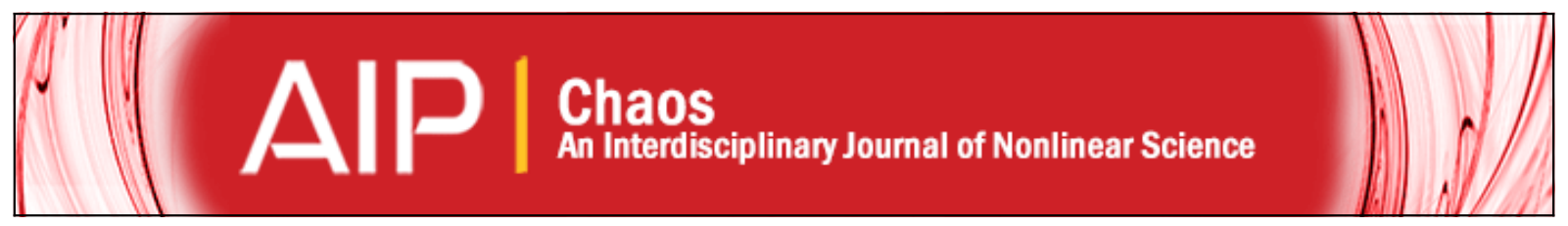

\title{
Winnerless competition between sensory neurons generates chaos: A possible mechanism for molluscan hunting behavior
}

Pablo Varona, Mikhail I. Rabinovich, Allen I. Selverston, and Yuri I. Arshavsky

Citation: Chaos: An Interdisciplinary Journal of Nonlinear Science 12, 672 (2002); doi: 10.1063/1.1498155

View online: http://dx.doi.org/10.1063/1.1498155

View Table of Contents: http://scitation.aip.org/content/aip/journal/chaos/12/3?ver=pdfcov

Published by the AIP Publishing

\section{Articles you may be interested in}

Four dimensional chaos and intermittency in a mesoscopic model of the electroencephalogram

Chaos 23, 023111 (2013); 10.1063/1.4804176

Mechanism, dynamics, and biological existence of multistability in a large class of bursting neurons

Chaos 20, 023118 (2010); 10.1063/1.3413995

Stochastic vagal modulation of cardiac pacemaking may lead to erroneous identification of cardiac "chaos"

Chaos 19, 028509 (2009); 10.1063/1.3141426

Dynamical phases of the Hindmarsh-Rose neuronal model: Studies of the transition from bursting to spiking chaos

Chaos 17, 043128 (2007); 10.1063/1.2818153

Topological analysis of chaos in neural spike train bursts

Chaos 9, 812 (1999); 10.1063/1.166455

\section{AlP Re-register for Table of Content Alerts}




\title{
Winnerless competition between sensory neurons generates chaos: A possible mechanism for molluscan hunting behavior
}

\author{
Pablo Varona ${ }^{\text {a) }}$ \\ Institute for Nonlinear Science, University of California, San Diego, 9500 Gilman Drive, La Jolla, \\ California 92093-0402 \\ and Grupo de Neurocomputación Biológica (GNB), Dpto. de Ingeniería Informática, \\ Universidad Autónoma de Madrid, 28049 Madrid, Spain \\ Mikhail I. Rabinovich, ${ }^{\text {b) }}$ Allen I. Selverston, ${ }^{\mathrm{c})}$ and Yuri I. Arshavsky ${ }^{\mathrm{d})}$ \\ Institute for Nonlinear Science, University of California, San Diego, 9500 Gilman Drive, La Jolla, \\ California 92093-0402
}

(Received 3 April 2002; accepted 12 June 2002; published 15 August 2002)

\begin{abstract}
In the presence of prey, the marine mollusk Clione limacina exhibits search behavior, i.e., circular motions whose plane and radius change in a chaotic-like manner. We have formulated a dynamical model of the chaotic hunting behavior of Clione based on physiological in vivo and in vitro experiments. The model includes a description of the action of the cerebral hunting interneuron on the receptor neurons of the gravity sensory organ, the statocyst. A network of six receptor model neurons with Lotka-Volterra-type dynamics and nonsymmetric inhibitory interactions has no simple static attractors that correspond to winner take all phenomena. Instead, the winnerless competition induced by the hunting neuron displays hyperchaos with two positive Lyapunov exponents. The origin of the chaos is related to the interaction of two clusters of receptor neurons that are described with two heteroclinic loops in phase space. We hypothesize that the chaotic activity of the receptor neurons can drive the complex behavior of Clione observed during hunting. (C) 2002 American Institute of Physics. [DOI: 10.1063/1.1498155]
\end{abstract}

The marine mollusk Clione is a predator lacking a visual system. How can it find its prey? Experimental observations during hunting behavior show that Clione moves randomly covering the three-dimensional space until it meets a prey. How does the small Clione nervous system organize such a search? We hypothesize that the origin of this hunting behavior is related to the complex (i.e., chaotic) dynamics of the mollusk's orientation sensory neural network. In the presence of prey, a specialized (hunting) neuron excites this network that, in the absence of prey, just sends a message to the motor system about the position of the body relative to the gravitational field. We present a model in which, under such excitation, the sensory network generates chaotic spatiotemporal patterns that mimic a random changing of direction in the gravitational field. As a result of receiving these chaotic patterns, the motor systems of Clione can produce the random-like hunting behavior. To our knowledge, this is the first report in which a network of competitive neurons with chaotic activity is used to drive a complex motor behavior.

\section{INTRODUCTION}

Clione is a marine mollusk that lives in cold waters. It swims by rhythmic movements of a pair of wings ${ }^{1}$ (Fig. 1,

\footnotetext{
${ }^{a}$ Electronic mail: pvarona@lyapunov.ucsd.edu

b)Electronic mail: mrabinovich@ucsd.edu

c)Electronic mail: aselverston@ucsd.edu

d)Electronic mail: yarshavs@ucsd.edu
}

upper panel). The direction of swimming is determined by the bending of the tail. Clione movements are controlled by the nervous system consisting of five pairs of ganglia. ${ }^{2}$ The motor neurons supplying wing and tail muscles are located in the pedal ganglia. ${ }^{1,2}$ When swimming, Clione maintains a vertical, head-up orientation. ${ }^{1,3}$ Any deviation from the vertical orientation produces a bending of the tail and changes in wing beating directed to the restoration of the initial position (Fig. 1, upper panel). Clione orientation in the gravitational field is determined by signals from the gravitational sensory organs, the statocysts. After abolition of inputs from the statocysts, Clione is not able to sustain any definite orientation. The statocyst is a sphere that contains a stone-like structure, the statolith, which moves inside the sphere under the effect of gravity (Fig. 2). The statocyst internal wall is lined with 9-11 statocyst receptor neurons (SRNs). ${ }^{4}$ The SRNs are mechanoreceptors responding to the pressure exerted by the statolith. The SRNs send axons to the cerebral ganglia where they affect the activity of cerebro-pedal interneurons controlling wing and tail motor neurons. ${ }^{3,5}$ Intracellular electrophysiological recordings from pairs of SRNs have shown that about $30 \%$ of them are coupled with inhibitory nonsymmetrical connections of different strength. ${ }^{3}$ Thus, we can conclude that the SRNs form a sensory neural network.

Clione is a predator; it feeds on a small mollusk, Limacina helicina. ${ }^{6}$ During hunting, Clione spatial orientation changes radically. The hunting behavior seems to be an intrincate search for prey. Clione swims in circles of small radius. The direction of the tail flexion is not constant during 

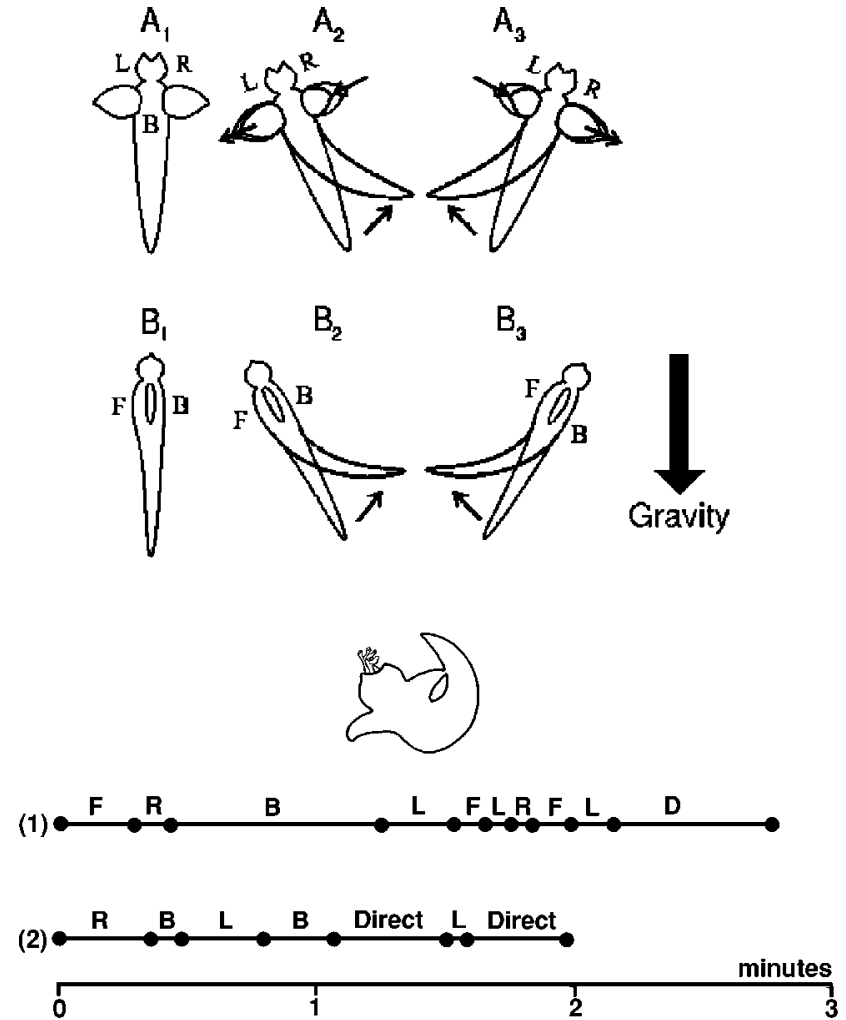

FIG. 1. Upper panel: behavioral characteristics of the postural orientation and equilibrium control in Clione. $\mathrm{A}_{1}, \mathrm{~B}_{1}$ : The most common orientation is the vertical one, with the head up (B, F, L, and R denote the back, front, left, and right aspects of the body). Deviation from this orientation will evoke correcting motor responses $\left(\mathrm{A}_{2}, \mathrm{~A}_{3}, \mathrm{~B}_{2}, \mathrm{~B}_{3}\right)$. Changes in the position of the tail and in the intensity of wing beating are marked with arrows (modified from Orlovsky et al.-Ref. 1). Bottom panel: spontaneous switches in spatial orientation of two Cliones ( 1 and 2) during hunting excited by a contact with the prey (shown above). Along the time axis, we indicate the periods of looping with the tail bent to the front (F), to the back (B), to the left (L), and to the right (R), as well as swimming downwards (D) and horizontally (direct); black spots indicate moments of changes in swimming direction (modified from Panchin et al.-Ref. 3).

hunting but changes in time in an irregular manner, which results in unpredictable changes in the plane of looping (Fig. 1 , bottom panel). This search behavior is triggered by the presence of Limacina. An important role in the organization of hunting behavior is played by a pair of large neurons located in the cerebral ganglia, the cerebral hunting neurons (CHNs) ${ }^{5,6}$ The CHNs control the activity of different neural networks involved in hunting behavior. Particularly, they exert an excitatory effect on the SRNs. Thus, the activity of the SRNs is determined not only by external sensory signals (orientation of the mollusk in the gravity field), but also by internal signals coming from the central nervous system through the CHNs.

In vitro experiments have shown that application of specific drugs to a preparation of the isolated nervous system can produce so-called "fictive random behavior." ${ }^{6}$ During "fictive behavior" the nervous system generates signals like those that command the actual hunting movements, although the muscles that realize these movements are absent. Particularly, during "fictive hunting behavior" the isolated nervous system can generate chaotic-like motor outputs to tail muscles. ${ }^{7}$ This fact is crucial for the building of a model of

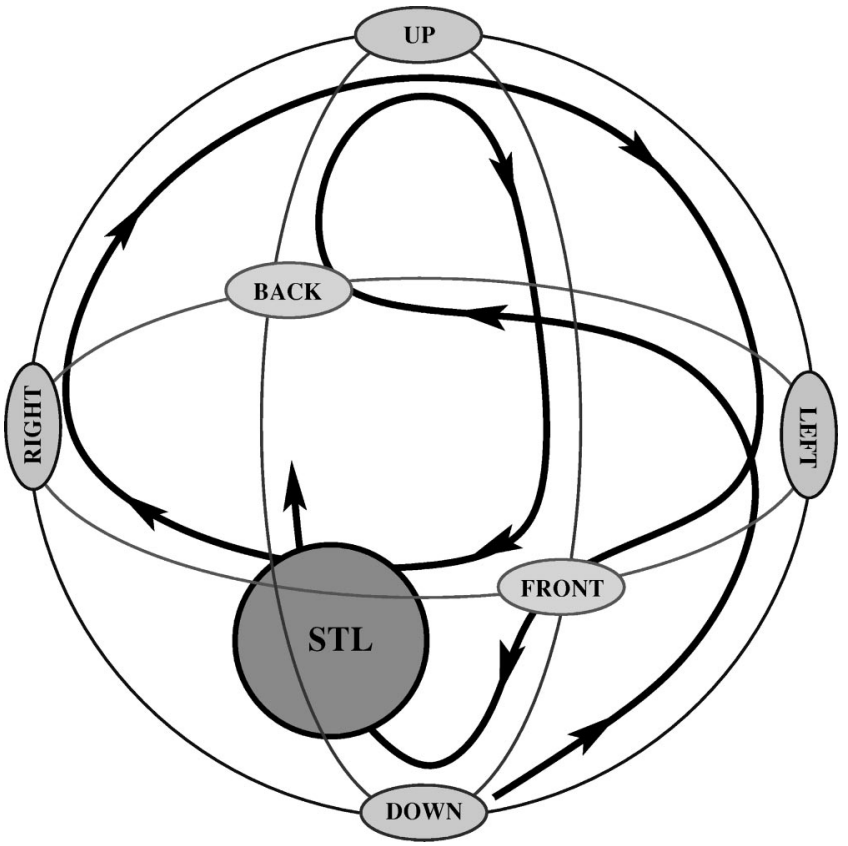

FIG. 2. Schematic representation of the statolith (STL) motion inside the statocyst, the gravity sensing organ of Clione. The receptor neurons (denoted as UP, DOWN, LEFT, RIGHT, BACK, FRONT) respond to the pressure exerted by the statolith (whose trajectory under the gravitational field is represented by the arrowed line). In the model explained in the text, the receptor excited by the statolith at a given time has the highest rate of activity. However, during hunting behavior the receptors receive an additional excitation from the hunting neuron, and this triggers the winnerless competition among the cells.

hunting behavior. Since the chaotic behavior is the result of the intrinsic activity of the isolated nervous system, it is not necessary to take into account in the model the motion of the mollusk body and the feedback from the peripheral motor system. This is correct at least if we wish to answer the first fundamental question: what is the origin of the apparent randomness during the hunting behavior? In this context, the construction of a complete model that takes into account the mechanical part of the problem is the second step.

The main goal of this paper is to show that, during hunting, the statocyst receptor neurons excited by the CHNs compete among each other and, as a result, they generate chaotic output signals. These spatiotemporal signals control motoneurons and can give rise to a chaotic changing of direction in the gravitational field.

\section{ORIGIN OF THE HUNTING DYNAMICS: THE MODEL}

The action of the statolith on the SRNs is represented schematically in Fig. 2. If no information about a prey (received by the chemical receptors) is present, and Clione is not in a "head up" position, a receptor neuron (other than D "down") is excited by the statolith. The information generated by this SRN arrives to the corresponding motoneurons that control the tail and wing movements. These motoneurons reestablish the habitual "head up" position of Clione's body. However, if the central hunting neuron receives a message from the chemo-sensors about the presence of a prey, the CHN sends excitatory inputs to the SRNs. The behavior 


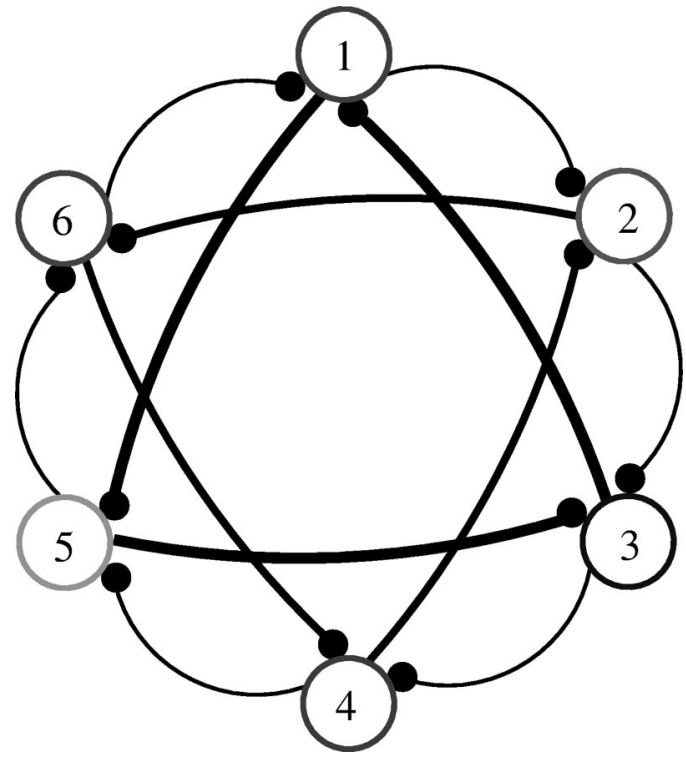

FIG. 3. Inhibitory connections used in the network of six statocyst receptor neurons (thicker traces mean stronger inhibition, see matrix 2).

of Clione in this case does not depend on the direction of the gravitational field. For the purpose of phenomenological modeling of statocyst "hunting" dynamics, we can neglect the statolith inertial dynamics as discussed before. Thus, we take into account the key point: the position of the mollusk's body uniquely depends on the message that SRNs are sending to the central neurons that produce the motor commands. As a starting point, we consider a statocyst with a SRN network of six neurons under the action of a single CHN excitation. Based on the physiological studies ${ }^{3}$ we suppose that, as a result of the CHN stimulation and when activated, all SRNs ("Left," "right," "back," "front," "down," and "up") send and receive two inhibitory signals to the rest of the network (see Fig. 3).

We have chosen the following Lotka-Volterra-type dynamics to describe the activity of the SRN network:

$$
\dot{a}_{i}=a_{i}\left(\sigma(\boldsymbol{H}, \boldsymbol{S})-\sum_{j=1}^{N} \rho_{i j} a_{j}+H_{i}(t)\right)+S_{i}(t),
$$

where $a_{i}>0$ represents the instantaneous spiking rate of the neurons, $H_{i}(t)$ represents the stimulus from the hunting neuron to neuron $i$, and $S_{i}(t)$ represents the action of the statolith on the receptor that is pressing. When there is no stimulus from the hunting neuron $\left(H_{i}=0, \forall i\right)$ or the statolith $\left(S_{i}=0, \forall i\right)$, then $\sigma(\boldsymbol{H}, \boldsymbol{S})=-1$ and all neurons are silent; $\sigma(\boldsymbol{H}, \boldsymbol{S})=1$ when the hunting neuron is active and/or the statolith is pressing one of the receptors.

The dynamical system (1) in the case $\sigma=1, \boldsymbol{H}(t)$ $=\boldsymbol{S}(t)=0$ is the familiar Lotka-Volterra system. ${ }^{8}$ The dynamics of the system is well known when the matrix $\rho_{i j}$ is symmetric $\left(\rho_{i j}=\rho_{j i}\right)$. In this case the autonomous system has a global Lyapunov function ${ }^{9,10}$ and every trajectory approaches one of the numerous possible equilibrium points. For example, if the inhibitory connections are identical, $\rho_{i j}$ $=\rho, \rho_{i i}=1$, this system has only one global attractor, e.g., $a_{i}=a_{0}=1 /[1+\rho(N-1)]$ for $\rho<1$, and $N$ attractors: $a_{i}$ $=a_{0}=1, a_{j \neq i}=0$ if $\rho>1$. No other attractors, e.g., limit cycles, or strange attractors are present in the system. The situation is much more complex and interesting when the inhibition is nonsymmetric: $\rho_{i j} \neq \rho_{j i}$ (see, for example, Fig. 3 ). A detailed analysis is only possible in the case $N=3$ (see Refs. 11-13). When $\rho_{i j}>1, \rho_{j i}<1$ there exists a heteroclinic orbit in the phase space of the system that consists of saddle points and one-dimensional separatrices connecting them. In some regions of the parameter space $\rho_{i j}$, such heteroclinic orbit (or limit cycle in its vicinity) is a global attractor. Suppose

$$
\rho_{i j}=\left(\begin{array}{ccc}
1 & \alpha_{1} & \beta_{1} \\
\beta_{1} & 1 & \alpha_{1} \\
\alpha_{3} & \beta_{3} & 1
\end{array}\right)
$$

and $0<\alpha_{i}<1<\beta_{i}$ and $\kappa_{i}=\left(\beta_{i}-1\right) /\left(1-\alpha_{i}\right)$. Then the heteroclinic orbit is a global attractor if $\kappa_{1} \kappa_{2} \kappa_{3}>1$, and the nontrivial fixed point $A\left(a_{1}^{0}, a_{2}^{0}, a_{3}^{0}\right)$ is a saddle point. If $\kappa_{1} \kappa_{2} \kappa_{3}=1$, this fixed point becomes neutrally stable and there exists a family of neutrally stable periodic solutions in the phase space. When $\kappa_{1} \kappa_{2} \kappa_{3}<1, A$ becomes a global attractor. The heteroclinic orbit exists but loses its stability. It is important to emphasize that in the case $\kappa_{1} \kappa_{2} \kappa_{3}>1$ a small perturbation is able to destroy the heteroclinic orbit and then a stable limit cycle appears in its vicinity. This limit cycle is characterized by a finite time period in contrast with the infinite time of motion along the heteroclinic loop (for example, see Ref. 14).

When $N>3$, even the autonomous system dynamics may be very complex. In particular, it is easy to imagine that depending on the distribution of the strength of the inhibitory connections, the system may have several different heteroclinic orbits. ${ }^{13}$ The simplest example is a system that consists of several independent or weakly connected triplets with strong interactions between neurons inside of each triplet as the one we are using here.

In our case $N=6$ and one possible set of values for the connection matrix $\rho_{i j} \neq 0$ is

$$
\begin{aligned}
& \rho_{1,3}=\rho_{3,5}=\rho_{5,1}=5, \\
& \rho_{4,6}=\rho_{2,4}=\rho_{6,2}=2, \\
& \rho_{1,6}=\rho_{2,1}=\rho_{3,2}=\rho_{4,3}=\rho_{5,4}=\rho_{6,5}=1.5, \\
& \rho_{1,1}=\rho_{2,2}=\rho_{3,3}=\rho_{4,4}=\rho_{5,5}=\rho_{6,6}=1 .
\end{aligned}
$$

A reasonable hypothesis about the inhibitory connections in the statocyst network that justifies this selection of values for $\rho_{i j}$ is the following. We have already mentioned that only about $30 \%$ of the recorded SRN pairs have inhibitory connections. Since it is known that the inhibitory connections are nonsymmetrical and have different strength (see Sec. I), let us assume that three of them are strong, three of them are moderate, and the rest of the connections are weak. If so, we can depict the topology of the connections inside the statocyst as in Fig. 3. 


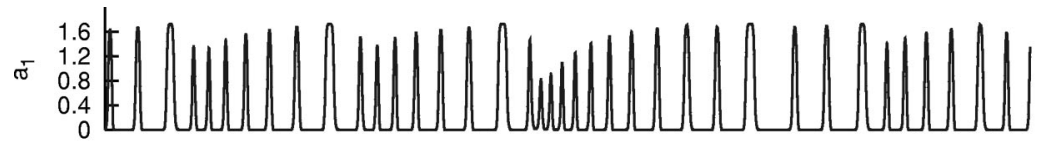

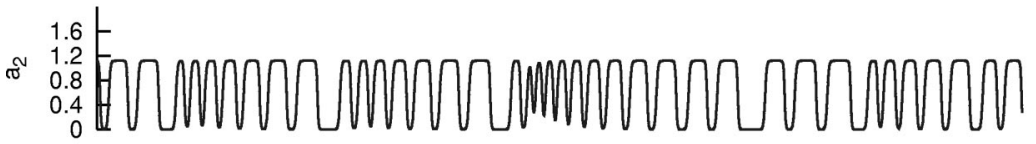
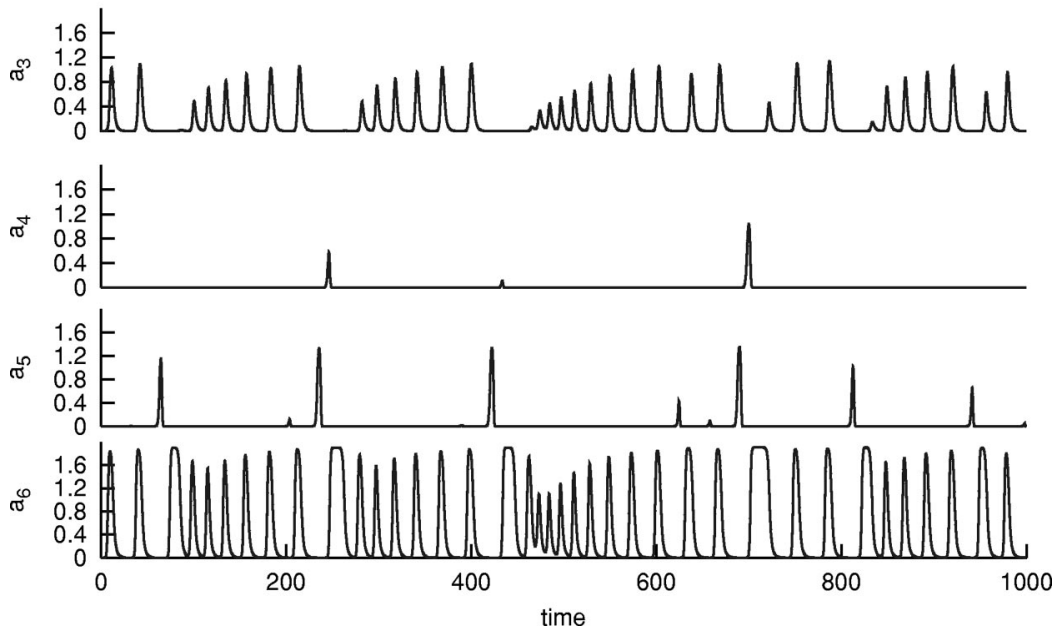

FIG. 4. Time series showing the chaotic switching of activities $a_{i}$ among the six receptor neurons induced by the action of the hunting neuron. Units are dimensionless in this model. See parameters used in the text.

\section{RESULTS}

When there is no activation of the sensory neurons from the hunting neuron, the effect of the statolith $\left(S_{i} \neq 0\right)$ in this model is to induce a higher rate of activity on one of the neurons (the neuron $i$ where it rests for a big enough $S_{i}$ value). We assume that this higher rate of activity affects the behavior of the motoneurons to organize the head up position. The other neurons are either silent or have a lower rate of activity and we can suppose that they do not influence the posture of Clione.

When the hunting neuron is active a completely different behavior arises. We assume that the action of the hunting neuron overrides the effect of the statolith and thus $S_{i}$ $\approx 0, \forall i$. The dynamical system (1) with the above-specified $\rho_{i j}$ values (see also Fig. 3) and with a stimuli from the hunt- ing neuron given, for example, by $H_{i}=(0.730,0.123,0.301$, $0.203,0.458,0.903)$ displays chaotic behavior. An illustration of the chaotic switching among the activities of the receptors can be seen in Fig. 4. Note how the periods in which each neuron is active evolve. The neurons keep the sequence of activation but the interval in which they are active is continuously changing in time. The power spectra for each of the neurons is depicted in Fig. 5.

Two three-dimensional projections of the attractor in the phase space are shown in Fig. 6. We calculated the Lyapunov exponents from the vector field (1) and found two positive Lyapunov exponents $\lambda_{1}=0.016$ and $\lambda_{2}=0.004$, and one zero exponent. The evolution in the calculation of the positive exponents is shown in Fig. 7.

Thus, in the presence of prey the SRN network generates
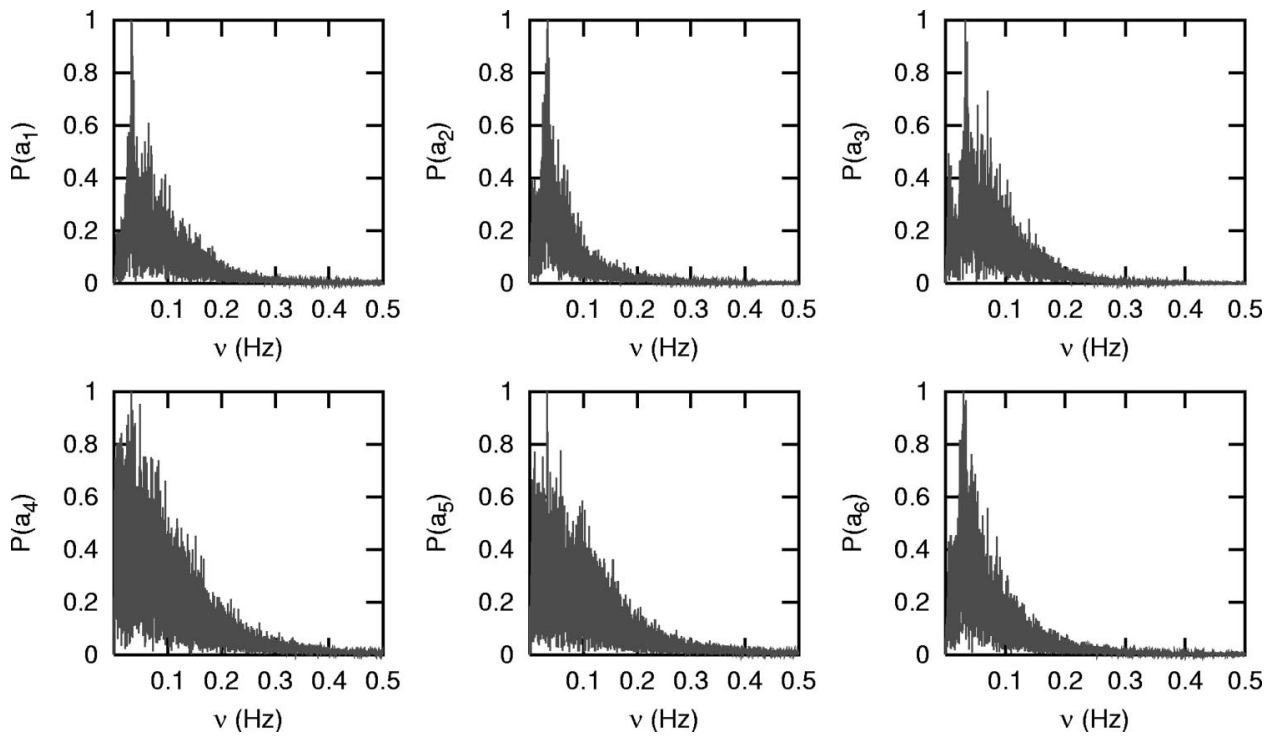

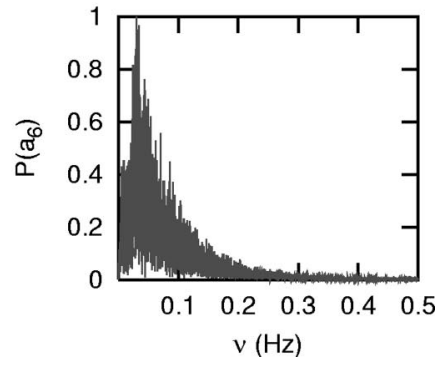

FIG. 5. Power spectrum for each of the neurons in the six receptor network under the action of the hunting neuron. 


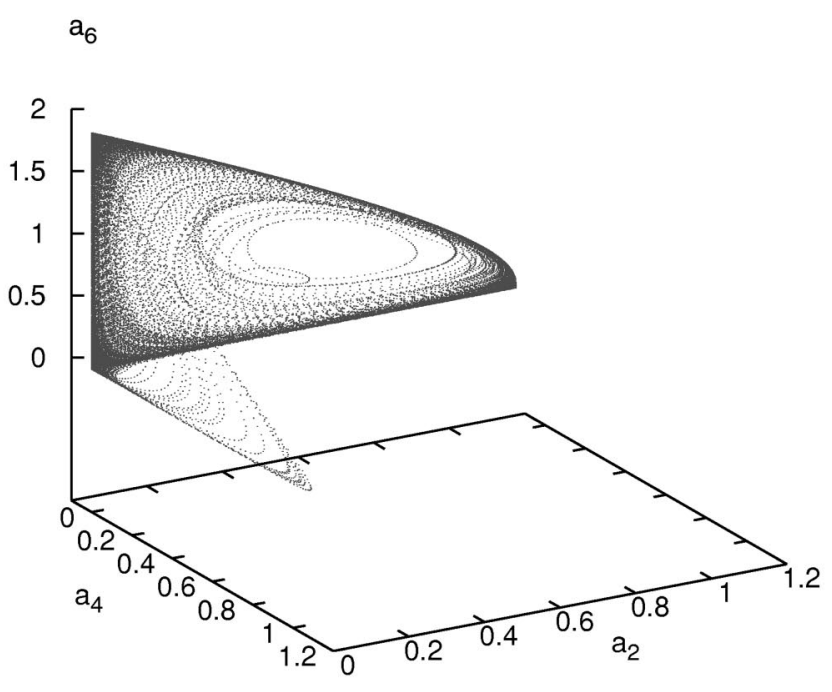

$a_{1}$

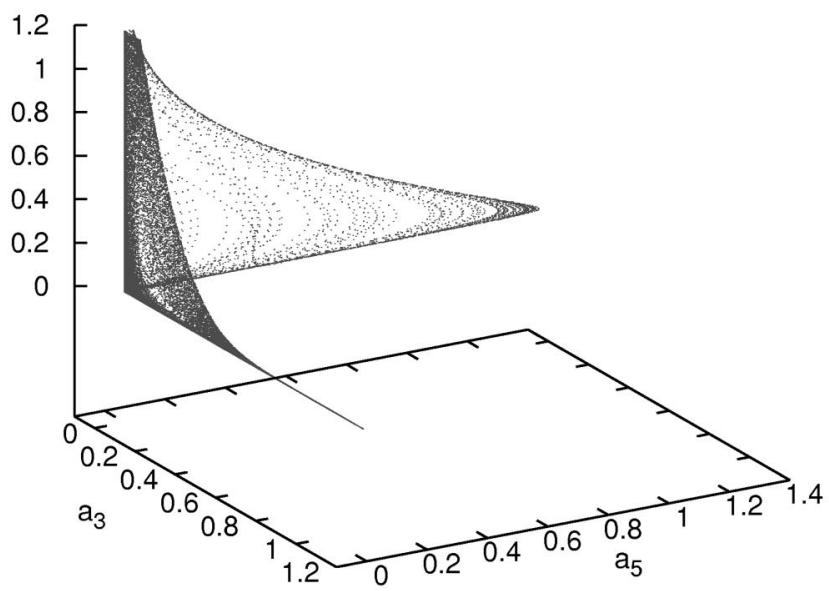

FIG. 6. Activity of the receptor neurons in the the two strongest connectivity triangles. Upper panel: three-dimensional $\left(a_{2}, a_{4}, a_{6}\right)$ projection of the phase space. Lower panel: $\left(a_{1}, a_{3}, a_{5}\right)$ projection.

new information, i.e., chaotic signals with positive Kolmogorov-Sinai entropy, which we hypothesize drives the motoneurons and, in fact, organizes the random-like hunting behavior of Clione.

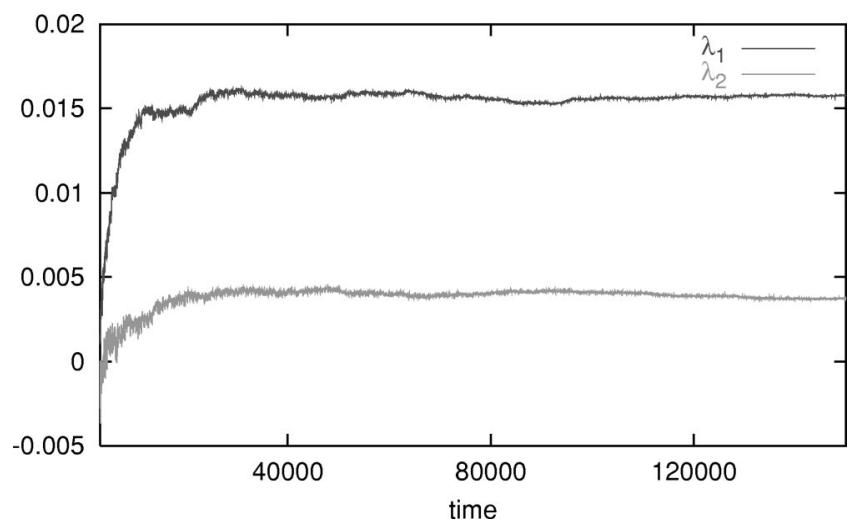

FIG. 7. Evolution in the calculation of the two positive Lyapunov exponents in the system of six receptor neurons under the action of the hunting neuron.
The origin of the chaoticity in this dynamical system can be explained in the following manner: Due to the diversity in the strengths of the inhibitory connections (see matrix 2 and the previous discussion) we may consider the complete network as two weakly coupled triangle networks. Independently, each of them has a closed heteroclinic orbit that becomes a limit cycle or a strange attractor under the action of a small perturbation (for example, any stimulus ${ }^{11}$ ). As we tested, the weak interaction of these two winnerless competitive triangles (nonlinear oscillators) generates the observed hyperchaos over a wide region of parameters $\rho_{i j}$ provided that the connections between neurons $i$ and $j$ are strongly nonreciprocal. For example, in the case $\left(\rho_{i j}-\rho_{j i}=\right.$ const $)$, it is necessary that $\left(\rho_{i j}+\rho_{j i}>2\right)$. Otherwise, the heteroclinic orbits in the individual triangles are not attractors and the dynamics of the system is completely different. However, the analyzed chaotic regime is structurally stable and a specific set of values for the connection matrix is not crucial.

\section{DISCUSSION}

The discussed neural ensemble is an example of nonsymmetric recurrent networks, which are typical for many basic neural circuits in the brain of vertebrates and invertebrates. The SRN network in Clione illustrates an important ability of nonsymmetric circuits to generate complex spatiotemporal patterns that control the behavior as a function of signals sensed from the environment. Such spatiotemporal patterns are not the modes of activity of a neural network programmed a priori, but activities that depend on stimuli in real time.

Neural networks with competitive dynamics are able to generate new information to answer a simple external signal. Such information can be used for the organization of complex activity and, in particular, chaotic-like behavior of animals as the one displayed by Clione during hunting. The quantitative value of this information is $I=K=\Sigma \lambda_{j}$, where $K$ is the Kolmogorov-Sinai entropy of the chaotic time series generated by the dynamical system $(I=0.02$ in our case). In this paper we have used a simple model to describe the activity of the receptor neurons of the gravity sensory organ. In spite of its simplicity, the model shows that competition among receptors excited by the hunting neuron generates chaotic activity that can organize the complex motion observed in this mollusk. Note that in this model the cooperative dynamics of the neurons are chaotic but they keep the sequence of activation when $a_{i}>0$. This is an interesting feature that could be used for the coordination of movements.

In our modeling we neglected two features of the statocysts: (i) the detailed intrinsic dynamics of the individual SRN neurons, and (ii) the statolith inertial dynamics. The critical question is: How do these factors influence the network dynamics, e.g., the appearance of chaotic behavior of the SRNs under the excitation of the hunting neuron? As we showed previously, the origin of the chaoticity in the SRN network is related to the on-off switching of the cooperative dynamics of the receptor neurons, which is guaranteed by the nonsymmetric inhibitory connections among the neurons excited by the hunting neuron. We have to point out that if the 
intrinsic dynamics of the elements of the network is more complex than the one analyzed here, even a symmetric network could generate chaotic spatiotemporal patterns. For example, a ring of an even number of oscillators with a strong enough nonlinearity has been reported to generate chaos. ${ }^{15,16}$

The details of the intrinsic neuron dynamics can be important for the calculation of quantitative characteristics of the chaotic time series but, as our preliminary modeling with Hodgkin-Huxley neurons shows, the qualitative features of the cooperative SRNs dynamics do not change. The role of the statolith can be crucial if the SRNs are below the spiking threshold (hyperpolarized). In this case, the excitation of specific SRN neurons (about one to three cells) by the statolith means the encoding of information about the position of the body of Clione relative to the gravity field. The situation becomes absolutely different when all of the SRN neurons are excited by the hunting neuron. The additional excitation of some neurons by the statolith cannot change the competitive interaction among SRNs qualitatively because of the nonsymmetric inhibition. Thus, the statolith motion has to be taken into account when determining the detailed boundary of the chaotic region in the control parameter space.

Chaotic dynamics in neural ensembles has been extensively discussed recently: from the mere report of its presence in different neural systems (isolated neurons and networks), to the analysis of the origin of the chaos and its functional role within brain function. ${ }^{17-20}$ Interestingly, chaotic activity does not often appear in collective behavior in recurrent networks, and it is often believed either to be an undesirable side effect of the complexity of the cells, or a proof of the complex dynamics of the neurons that shows up in transient behavior and not during normal activity. Here we have reported how a network of competitive neurons organizes chaotic activity that could be useful in driving a complex behavior.

\section{ACKNOWLEDGMENTS}

We thank Ramón Huerta, Rafael Levi, and Henry Abarbanel for many useful discussions about the model. Support for this work came from NIH Grant No. 2R01 NS3802205A1. P.V. acknowledges support from MCT BFI2000-0157. M.R. acknowledges support from U.S. Department of Energy Grant No. DE-FG03-96ER14592.

${ }^{1}$ G. N. Orlovsky, T. G. Deliagina, and S. Grillner, Neuronal Control of Locomotion. From Mollusc to Man (Oxford University Press, New York, 1999).
${ }^{2}$ Y. I. Arshavsky, I. N. Beloozerova, G. N. Orlovsky, Y. V. Panchin, and G. A. Pavlova, "Control of locomotion in marine mollusc Clione limacina. I. Efferent activity during actual and fictitious swimming," Exp. Brain Res. 58, 255-262 (1985).

${ }^{3}$ Y. V. Panchin, Y. I. Arshavsky, T. G. Deliagina, L. B. Popova, and G. N. Orlovsky, "Control of locomotion in marine mollusk Clione limacina. IX. Neuronal mechanisms of spatial orientation," J. Neurophysiol. 75, 1924 1936 (1995).

${ }^{4}$ T. P. Tsirulis, "The fine structure of the statocyst in the gastropod mollusc Clione limacina," Zh. Evol. Biokhim. Fiziol. 10, 181-188 (1974) (in Russian).

${ }^{5}$ Y. V. Panchin, L. B. Popova, T. G. Deliagina, G. N. Orlovsky, and Y. I. Arshavsky, "Control of locomotion in marine mollusk Clione limacina. VIII. Cerebro-pedal neurons," J. Neurophysiol. 73, 1912-1923 (1995).

${ }^{6}$ Y. I. Arshavsky, T. G. Deliagina, G. N. Gamkrelidze, G. N. Orlovsky, Y. V. Panchin, and L. B. Popova, "Pharmacologically-induced elements of feeding behavior in the pteropod mollusc Clione limacina. II. Effect of physostigmine," J. Neurophysiol. 69, 522-532 (1993).

${ }^{7}$ M. I. Rabinovich, P. Varona, Y. I. Arshavsky, and A. I. Selverston, "Dynamical origin of the random-like hunting behavior of the marine mollusk Clione," Soc. Neurosci. Abs. 27, 943.13 (2001).

${ }^{8}$ N. MacDonald, Trees and Networks in Biological Models (Wiley, New York, 1983).

${ }^{9}$ M. A. Cohen and S. Grossberg, "Absolute stability of global pattern formation and parallel memory storage by competitive neural networks," IEEE Trans. Syst. Man Cybern. 5, 815-826 (1983).

${ }^{10}$ J. J. Hopfield, "Neural networks and systems with emergent selective computational abilities," Proc. Natl. Acad. Sci. U.S.A. 79, 2554-2558 (1982).

${ }^{11}$ V. S. Afraimovich, S. B. Hsu, and H. E. Lin, "Chaotic behavior of three competing species of May-Leonard model under small periodic perturbations," Int. J. Bifurcation Chaos Appl. Sci. Eng. 11, 435-447 (2001).

${ }^{12}$ C. W. Chi, S. B. Hsu, and L. I. Wu, "On the asymmetric May-Leonard model of three competing species," SIAM (Soc. Ind. Appl. Math.) J. Appl. Math. 58, 211-226 (1998).

${ }^{13}$ M. I. Rabinovich, A. Volkovskii, P. Lecanda, R. Huerta, H. D. I. Abarbanel, and G. Laurent, "Dynamical encoding by networks of competing neuron groups: Winnerless competition," Phys. Rev. Lett. 87, 068102 (2001).

${ }^{14}$ M. I. Rabinovich, A. B. Ezersky, and P. D. Weidman, The Dynamics of Patterns (World Scientific, Singapore, 2001).

${ }^{15}$ J. J. Collins and I. Stewart, "Hexapodal gaits and coupled nonlinear oscillator models," Biol. Cybern. 68, 287-298 (1993).

${ }^{16}$ A. V. Gaponov-Grekhov and M. I. Rabinovich, Nonlinearities in Action (Springer, Berlin, 1992).

${ }^{17}$ T. Elbert, W. J. Ray, Z. J. Kowalik, J. E. Skinner, E. E. Graf, and N. Birbaumer, "Chaos and physiology: Deterministic chaos in excitable cell assemblies," Physiol. Rev. 74, 1-47 (1994).

${ }^{18} \mathrm{H}$. Hayashi and S. Ishizuka, "Chaotic nature of bursting discharges in the Onchidium Pacemar neuron," J. Theor. Biol. 156, 269-291 (1992).

${ }^{19}$ P. Varona, J. J. Torres, R. Huerta, H. D. I. Abarbanel, and M. I. Rabinovich, "Regularization mechanisms of spiking-bursting neurons," Neural Networks 14, 865-875 (2001).

${ }^{20}$ R. Huerta, P. Varona, M. I. Rabinovich, and H. D. I. Abarbanel, "Topology selection by chaotic neurons of a pyloric central pattern generator," Biol. Cybern. 84, L1-L8 (2001). 